\title{
Cyclotron Resonance and Quasiparticles
}

\author{
Marvin L. Cohen \\ Department of Physics, University of California, Berkeley, California 94720-7300 \\ Materials Sciences Division, Lawrence Berkeley National Laboratory, One Cyclotron Road, Berkeley, California
}

94720

\begin{abstract}
This introductory paper contains personal perspectives about the importance of cyclotron resonance in forming our modern view of solids. The papers following this one will discuss the discovery, refinements, and some of the latest developments. Although I will touch on some of these subjects, I leave the details to the other authors and in the main focus on the conceptual impact of the work. I propose that it was experiments based on cyclotron resonance which established the quasiparticle concept.
\end{abstract}

In a mature field like condensed matter physics, a large effort is devoted to testing the newest ideas. Although one is aware that traditional models and concepts are being used to think about the newest ideas, it isn't common to ask about the foundations, conceptions, or assumptions about our field until something doesn't fit. We just use these concepts. For example, electronic band structures and effective masses of electrons and holes in solids are standard tools or concepts for interpreting experiments. Another case is the quasiparticle picture which assumes that electrons can act as "dressed" particles. Examples of quasiparticles include the polaron and the concept of a hole. Both are very convenient and useful ways of interpreting experimental data.

Many younger researchers who use these ideas daily may feel that the concepts above are "obvious and natural" and have been here forever. However, when one views a material as a collection of interacting atoms, it's almost a leap of faith to embrace the elementary excitation picture of a solid with its collective excitations and quasiparticles. It's easy to state or demonstrate mathematically that lattice vibrations can be viewed as phonons and collective electronic motion can be represented by plasmons. Sometimes we lose touch with the fact that these and other boson-like excitations such as magnons are mental constructions. Similarly for the fermion-like excitations, one cannot make a beam of holes propagating across an empty tube, but to many of us, holes exist. So it is important to measure response functions such as dielectric functions and magnetic susceptibilities and show experimentally that they can be interpreted for the most part in terms of boson and fermion excitations such as the collective excitations and quasiparticles commonly listed in descriptions of the elementary excitation model of solids. One can then argue that since they describe physical behavior, they exist.

Similarly, the concept of energy bands can be established from an interacting atom's picture where atomic energy levels are perturbed and bands of allowed states are formed; however the calculated dispersion of the bands and the concept that individual particle-like excitations will occur near band edges are not obvious. In a sense some of this can be included with the general idea of a quasiparticle concept. The effective mass, in principle, can contain both band structure effects and electron mass renormalization arising from interactions of the electron with other elementary excitations.

The measurement of effective masses using cyclotron resonance gives information about the interaction of an electron with the periodic potential and also about the formation of electronic quasiparticles such as polarons. In addition, band structure effects such as anisotropy, spin-orbit splittings, and band dispersions can be measured to test the calculations and the underlying conceptual models. 
In effect, I propose that it was experiments based on cyclotron resonance that established the quasiparticle concept. If an electron reacts to a probe as if it were an electron with charge e but mass $\mathrm{m}^{*}$, not equal to the free electron mass, then it is acceptable to view it as such. In the fractional quantum Hall effect, theory proposed that we think of the quasiparticles as electrons having fractional charges. And again, experiments are consistent with this picture, so nowadays having discussions of electrons with modified masses and charges are commonplace.

When cyclotron resonance was first reported [1], band structure calculations and the measurements of band structure "effects" were not highly developed areas. Even for $\mathrm{Ge}$ and $\mathrm{Si}$, knowledge about electronic structure determinations was primitive. As C. Kittel [2] has described, the plan to observe cyclotron resonance was a "prime objective" of the Berkeley solid-state physics group. This classic experiment was done on borrowed equipment including a 10-inch magnet from E.O. Lawrence, recycled Navy submarine batteries, Ge from Sylvania and Westinghouse, liquid helium from Shell Laboratories, and war surplus microwave gear. Theoretical input was essential. The avoidance of having the magnetoplasma resonance (which was also observed in these studies) mask cyclotron resonance was a major concern. Producing carriers with long lifetimes was essential.

The interpretation of the two measured effective masses [1] in $\mathrm{Ge}$ as arising from two sets of holes led to the spin-orbit analysis and the split valence band pictures viewed in terms of light and heavy holes. I won't discuss the main application of this model since much of the work for studying ionization energies of shallow impurities and other semiconductor properties is well documented. My focus here is that it was clear that in the analysis of the experiment the concept of heavy and light holes meant that one could view excitations near the valence band maximum as if they were particles. The independent particles model of band edges was therefore implied in the interpretation of these data.

The point made above that excitations of states near band edges can be treated as particles, or more correctly as quasiparticles, is also demonstrated in the MIT work where the measured anisotropy of band states was used to verify the band pictures. For example, in $\mathrm{Si}$, where the conduction band edge is on the $\Delta$ symmetry line, by changing the direction of the static magnetic field relative to the oscillating EM field, it was possible to determine the anisotropic nature of these electronic states. It was established that each of the six valleys of the Si conduction band could be described as having a longitudinal mass $m=0.98 m$ and a transverse mass $m_{t}=0.19 m$. Again, to view the data in terms of excitations of quasiparticles with masses differing by a factor of five depending on the direction of the magnetic field is a further demonstration of the breadth of this concept.

With proper choices of crystals and conditions, the spectroscopic nature of this experimental approach was exploited. If one views the physical picture in terms of photon-induced transitions of electrons between Landau levels resulting from a static field, then the exciting frequency can be varied and used to probe the electronic band structure. For example, the infrared cyclotron resonance measurements done early at NRL can be viewed as measurements of the optical response function for these photon-induced electronic transitions between Landau levels. Again, band structure and quasiparticle notions are important.

As mentioned earlier, not only does the measured effective mass contain band structure effects, there are also contributions of mass renormalization arising from electron-phonon interactions and electronelectron contributions through virtual excitations of plasmons and electron-hole pairs. The early work by Landau [3] attempted to explain F-centers using what we now consider a polaron concept. He proposed the idea of a self-trapped electron with the trap arising from the interaction of the electron with the polarization caused by the ions in the crystal. This was followed by the consideration of a model [4] where the electron and its polarization potential move together through the lattice. This is an example of a strongly coupled polaron.

Fröhlich [5] later gave a quantitative treatment of electron scattering in ionic crystals and then applied field-theoretic methods [6] for this problem when he was attempting to construct a theory of superconductivity. Because the polaron is a prototype model for a fermion interacting with a scalar boson, it attracted researchers whose major interest was in particle physics [7].

Since cyclotron resonance is the principle experiment for measuring effective masses, it is of central importance in establishing the validity of these theories. Below, I describe briefly the most illustrative case related to polarons and the influence of the related concepts on other areas such as the physics of metals and superconductivity.

In the simplest weak binding model of a single electron in a polar crystal interacting with a 
longitudinal optical (LO) phonon, the theoretical results are mostly straightforward. This is the Fröhlich problem where one calculates the lowest order electron self-energy diagram to get the self energy shift and the effective mass change arising from the scattering of the electron by virtual LO phonons. The result for the energy to order $\mathrm{k}^{2}$ is

$$
E(k)=-\alpha \omega+\frac{{ }^{2} k^{2}}{2 m_{b}}(1-\alpha / 6)
$$

where the first term is the energy shift given in terms of the dimensionless Fröhlich electron-phonon coupling constant $\alpha$ and the LO phonon frequency $\omega$. The second term demonstrates the effective mass $m^{*}$ by associating the coefficient of the square of the wave vector $\mathrm{k}$ with a term

$$
m^{*}=\frac{m_{b}}{1-\alpha / 6}
$$

to express the enhancement of the band mass $m_{b}$. For small $\alpha$, this is usually written as

$$
m^{*}=m_{b}(1+\alpha / 6)
$$

Although this is just about the simplest model for interpreting the measured $\mathrm{m}^{*}$ in a cyclotron resonance experiment, it is often applied to much more complicated cases without proper justification. This assumption for many experiments has been recently challenged [8]. In particular, it can be argued that once more than one electron is excited, screening effects and other considerations must be handled carefully.

It is interesting that the proper description of multiple electrons complicates the interpretation of cyclotron resonance data since the concerns of the authors of reference (1) was focused so strongly on plasma effects and the resulting magnetoplasma resonance. It was this concern and the skin depth problem which were so discouraging when cyclotron resonance experiments in metals were first contemplated. These problems were eventually overcome by changing the geometry and allowing the EM field to accelerate the electrons when they were within the skin depth.

Although the term polaron was defined to describe the case of a single electron in an ionic insulator coupled to an LO phonon, its use has been expanded to describe electronic quasiparticles in insulators and semiconductors with arbitrary coupling to phonons be they acoustical or optical. In addition the notion and name has been used for electrons in metals. Here we don't expect to observe a self energy shift near a band gap described by Eq. (1), but rather a "wrinkle" in the $\mathrm{E}(\mathrm{k})$ curve at the Fermi energy with an energy width of order the phonon energy. The shape of the wrinkle gives an increased mass $m^{*}$ at the Fermi energy

$$
m^{*}=m_{b}(1+\lambda)
$$

in analogy with Eq. (3). The electron-phonon parameter $\lambda$ is analogous to the Fröhlich $\alpha$. This is often called a "polaron effect". Despite the fact that much of the physics of mass renormalization in metals is very different from the Fröhlich problem, the descriptive words are often borrowed from the latter.

For many metals, the Landau theory of Fermi liquids allows quasiparticle pairing through phonon exchange with an electron-phonon coupling parameter $\lambda$. So once more the interpretation of experiments in terms of excitations of quasiparticles resembles the approach used to explain cyclotron resonance.

In the more modern field theoretic versions of $\mathrm{BCS}$, a self-consistent approach is more appropriate than just considering the pairing of normal metal electrons or quasielectrons. In fact, although it is common to use the $\lambda$ as defined in Eq. (4) to pair electrons for superconductivity, things can be more complex. Using heat capacity measurements or cyclotron resonance measurement to determine $\mathrm{m}^{*}$ along with calculations of $m_{b}$, one can extract $\lambda$ and then use this value of $\lambda$ to calculate the superconductivity transition temperature $T_{c}$. Generally, this works fairly well, but fails completely for $\mathrm{MgB}_{2}$. This failure brings in interesting future possibilities for cyclotron resonance measurement since the resolution [9] of this dilemma is that the Fermi surface has distinct regions. These regions can be roughly grouped into two parts. The $\lambda$ 's associated with these two parts are different and the electron phonon pairing parameters differ from the mass enhancement $\lambda$ 's creating two superconducting gaps. Systems of this type are of interest physically and as potentially higher $T_{c}$ superconductors. Cyclotron resonance measurements of $\mathrm{m}^{*}$ for different parts of the Fermi surface could help in a detailed study of these interesting systems. A constraint in this particular case is that high quality single crystals are difficult to make.

Two-dimensional systems have been the objects of intense study since high mobility samples have become available. Again cyclotron resonance experiments have been used to determine $\mathrm{m}^{*}$ 's for electron and hole excitations in heterostructures such 
as GaAs/AlGaAs and inversion layers on $\mathrm{Si}$. The quantum Hall effect and the fractional quantum Hall effect observed in heterojunctions are examples of correlated electronic systems with their related quasiparticles as described earlier. Newer results on the so called "microwave induced zero-resistance state" will be described in one of the following papers. Here, I emphasize again the concepts described above are applicable.

In going to two dimensions and even to quasi-one dimensional systems such as carbon and boron nitride nanotubes, we still retain the quasiparticle picture much of the time to interpret measured data. Reduced dimensionality brings confinement effects and symmetry often plays a more dominant role. For example, two metallic nanotubes in contact may not allow a current to flow through their contact region because of symmetry constraints. Moving to quasizero dimensional systems such as quantum dots and molecules, again confinement and symmetry are central considerations. However, for most of the theoretical studies of reduced dimensional systems, the quasiparticle picture is used, and again we owe a debt to the cyclotron resonance studies which established this concept. The applications to band theory, optical properties, impurity states, excitons, superconductivity, and transport in $3 \mathrm{D}, 2 \mathrm{D}$, quasi-one $\mathrm{D}$ and quasi-zero $\mathrm{D}$ systems gives us a vast amount of information about macro, micro, and nano systems.

In the so-called "standard model of solids" which works for a broad class of crystals, the valence electrons are viewed as interacting with positive cores in a manner that can be described [10] using pseudopotentials and density functional theory. Excitations from the ground-state are computed with an "elementary excitation" view as described earlier. Experiments such as cyclotron resonance and angular resolved photoelectron spectroscopy show that this view works for electronic quasiparticle excitations and that the electronic band structures calculated using the "standard model" are essentially correct and predictive.

It is likely that cyclotron resonance experiments will continue as a valuable resource for establishing the "standard model" and quasiparticle picture and for finding examples where they don't apply.

\section{ACKNOWLEDGMENT}

I would like to acknowledge Professor C. Kittel for discussions. This work was supported by National Science Foundation Grant No. DMR00-87088 and by the Director, Office of Science, Office of Basic Energy Sciences, Division of Materials Sciences and Engineering, U.S. Department of Energy under Contract No. DE-AC03-76SF00098.

\section{REFERENCES}

1. Dresselhaus, G., Kip, A. F., and Kittel, C., Phys. Rev. 92, 827 (1953).

2. Kittel, C. in Fundamentals of Semiconductors by P.Y. $\mathrm{Yu}$ and M. Cardona, Berlin, Springer-Verlag, 1996, p. 547.

3. Landau, L. D., Phys. Z. Sowjetunion 3, 644 (1933).

4. Landau, L. D. and Pekar, S., Zh. Eksp. i Teor. Fiz. 16, 341 (1946).

5. Fröhlich, H., Proc. Roy. Soc. A160, 230 (1937).

6. Fröhlich, H., Pelzer, H., and Zienau, S., Phil. Mag. 41, 221 (1950).

7. Feynman, R. P., Phys. Rev. 97, 660 (1955).

8. Faugeras, C., Martinez, G., Riedel, A., Hey, R., Friedland, K. J., and Bychkov, Yu., Phys. Rev. Lett. 92, 107403 (2004).

9. Choi, H.J., Roundy, D., Sun, H., Cohen, M.L., and Louie, S.G., Nature 418, 758 (2002).

10. Cohen, M.L., Physica Scripta T1, 5 (1982). 